\title{
Analysis of gender-specific associations between aldehyde dehydrogenase 2 (ALDH2) rs671 genetic polymorphisms and serum uric acid levels in Han Chinese
}

\author{
Jie Wu ${ }^{1 \#}$, Chaochao $\mathrm{Ma}^{1 \#}$, Jie $\mathrm{Yi}^{1}$, Yu Chen ${ }^{1}$, Ali Ye ${ }^{1}$, Lingjun Kong ${ }^{1}$, Ling Qiu ${ }^{1}$, Tengda Xu ${ }^{2}$ \\ ${ }^{1}$ Department of Laboratory Medicine, Peking Union Medical College Hospital, Peking Union Medical College \& Chinese Academy of Medical \\ Science, Beijing, China; ${ }^{2}$ Department of Health Care, Peking Union Medical College and Chinese Academy of Medical Science, Beijing, China \\ Contributions: (I) Conception and design: J Wu, C Ma; (II) Administrative support: L Qiu, T Xu; (III) Provision of study materials or patients: J Yi, Y \\ Chen, A Ye, L Kong; (IV) Collection and assembly of data: J Wu, C Ma; (V) Data analysis and interpretation: C Ma, J Wu; (VI) Manuscript writing: \\ All authors; (VII) Final approval of manuscript: All authors. \\ \#These authors contributed equally to this work. \\ Correspondence to: Prof. Ling Qiu. Department of Laboratory Medicine, Peking Union Medical College Hospital, Peking Union Medical College \\ \& Chinese Academy of Medical Science, Beijing 100730, China. Email: lingqiubj@163.com; Prof. Tengda Xu. Department of Health Care, Peking \\ Union Medical College and Chinese Academy of Medical Science, Beijing 100730, China. Email: Xutd@pumch.cn.
}

Background: Serum uric acid (SUA) is influenced by lifestyle and genetics, and unbalanced SUA levels are linked to various common disorders. While the aldehyde dehydrogenase 2 (ALDH2) rs671 polymorphism appears to be associated with SUA levels, the evidence remains inconclusive. The aim of this study was to examine the distribution of the ALDH2 rs671 polymorphism among Han Chinese in Beijing and determine the association between this polymorphism and SUA.

Methods: A total of 6,461 randomized healthy individuals were included in the study. Biochemical indicators were tested and ALDH2 rs671 polymorphism testing was conducted for subjects enrolled in the study. The distribution of the ALDH2 rs671 polymorphism and the relationship between genotype and the levels of serum lipids and uric acid (UA) were analyzed.

Results: The ALDH2 rs671 genotype frequencies were 68.1\% (G/G), 29.3\% (G/A), and 2.6\% (A/A). There was no significant difference in allele distribution between males and females. In males, different ALDH2 genotypes exhibited significant differences in several biochemical analytes, including body mass index (BMI), blood glucose (Glu), total cholesterol (TC), high-density lipoprotein cholesterol (HDL-C), UA, glutamyl transpeptidase (GGT), and creatinine $(\mathrm{Cr})(\mathrm{P}<0.05)$. No such differences were found in females. SUA levels in G/A and A/A-carrying males were significantly lower than those of G/G-carrying males. The effect of the ALDH2 polymorphism on UA was still significant after further adjustment for factors including BMI, Glu, TC, HDL-C, Cr, and GGT.

Conclusions: The ALDH2 polymorphism is related to SUA in Beijing males, and A allele-carrying males have lower SUA levels.

Keywords: Aldehyde dehydrogenase 2 (ALDH2); gene polymorphism; uric acid (UA)

Submitted Oct 25, 2020. Accepted for publication Feb 28, 2021.

doi: $10.21037 / \mathrm{atm}-20-7113$

View this article at: http://dx.doi.org/10.21037/atm-20-7113

(c) Annals of Translational Medicine. All rights reserved. 


\section{Introduction}

Uric acid (UA) and urate are the end products of purine nucleoside degradation, and the UA pool in vivo is determined by the balance of its synthesis and clearance. It is well known that hyperuricemia is a major risk factor for gout (1). Furthermore, increased UA levels may be a potential risk factor for metabolic syndrome, cardiovascular disease, and type 2 diabetes (2-5). The level of serum UA (SUA) is not only affected by environmental factors but also by genetic factors. Genome-wide association studies (GWAS) of SUA have confirmed that multiple gene variants are associated with SUA levels (6-8). Variations in SLC2A9 and $A B C G 2$ remain the major genetic determinants of SUA (9), and have been validated in the Chinese population (10). Our previous study revealed that apolipoprotein $\mathrm{E}(A p o E)$ gene polymorphism is associated with SUA levels in a Chinese population and subjects with an $\varepsilon 2$ allele have higher levels of SUA (11).

The aldehyde dehydrogenase $2(A L D H 2)$ gene, located in the $12 \mathrm{q} 24$ chromosomal region, encodes the mitochondrial ALDH2 protein, which is one of the most important enzymes in human alcohol metabolism. The most wellknown substrate of ALDH2 is acetaldehyde. In the human body, ALDH2 oxidizes acetaldehyde, an intermediate metabolite of ethanol, to acetic acid, which then enters the tricarboxylic acid cycle. A single nucleotide polymorphism (SNP) site is present on the 12th exon of ALDH2 (rs671), in which guanine $(\mathrm{G})$ is replaced by adenine (A), resulting in the conversion of the encoded glutamate to lysine. The mutation results in decreased or even a complete loss of ALDH2 activity. The ALDH2 (G/A) heterozygote displays only $10 \%$ to $45 \%$ individual enzyme activity, while the variant-type homozygote (A/A) has only $1 \%$ to $5 \%$ activity. It has been shown that subjects with the A allele have a reduced capacity to eliminate acetaldehyde and typically have alcohol-induced "flushing" syndrome which includes flushing, nausea, and rapid heart rate (12). Allele A of the defective $A L D H 2$ polymorphism (rs671) is highly prevalent in East Asian countries, such as China, Japan, Korea, Mongolia, and Indochina. The highest frequency of this variant has been reported in southeast China (13).

In addition to its effect on alcohol consumption, rs671 polymorphism of $A L D H 2$ is associated with hypertension, dyslipidemia, type 2 diabetes mellitus, and cardiovascular diseases $(14,15)$. Recent studies have suggested that $A L D H 2$ is associated with SUA levels and gout susceptibility $(16,17)$. In addition, a GWAS study identified novel loci that aggravate asymptomatic hyperuricemia into gout. One of them, rs671 of $A L D H 2$, was identified as a gout locus in this study for the first time (18). These previous studies were performed in other population groups, used small sample sizes, or did not report on sex-linked differences. Therefore, more studies are needed to explore the correlation between $A L D H 2$ and SUA and any potential gender differences. In this study, we explored the relationship between $A L D H 2$ polymorphism and SUA level in both the genders. We present the following article in accordance with the MDAR checklist (available at http://dx.doi.org/10.21037/atm-207113).

\section{Methods}

\section{Study population}

The present study included 6,461 subjects who underwent health checkups in Peking Union Medical College Hospital between January 2014 and August 2017. These subjects were randomly included in our study, with an average age of $47.5 \pm 8.77$ years, and included 4,123 males with an average age of $47.4 \pm 8.76$ years and 2,338 females with an average age of $47.6 \pm 8.78$ years. Participants with known systemic diseases such as hypertension, diabetes mellitus, renal or liver disease, cardiovascular disease, gastrointestinal disease, pulmonary disease, or cancer were excluded. The schedule describing participant enrollment is presented in Figure 1.

\section{Ethics approval}

The study was performed in accordance with the Helsinki Declaration (as revised in 2013) and was approved by the Ethics Committee of Peking Union Medical College \& Chinese Academy of Medical Sciences, Peking Union Medical College Hospital (ethical approval document number: S-K972). Since the data used in this study are removed privacy, it will not pose any risk to the subjects and cannot trace it back to subjects. Therefore, informed consent was not required.

\section{Clinical laboratory tests}

All procedures were performed following a 9-12-h overnight fast. Blood pressure (BP) was measured after the participant had rested for at least $10 \mathrm{~min}$. Blood was drawn from the antecubital vein of the arm and levels of glucose (Glu), total cholesterol (TC), triglycerides (TG), 


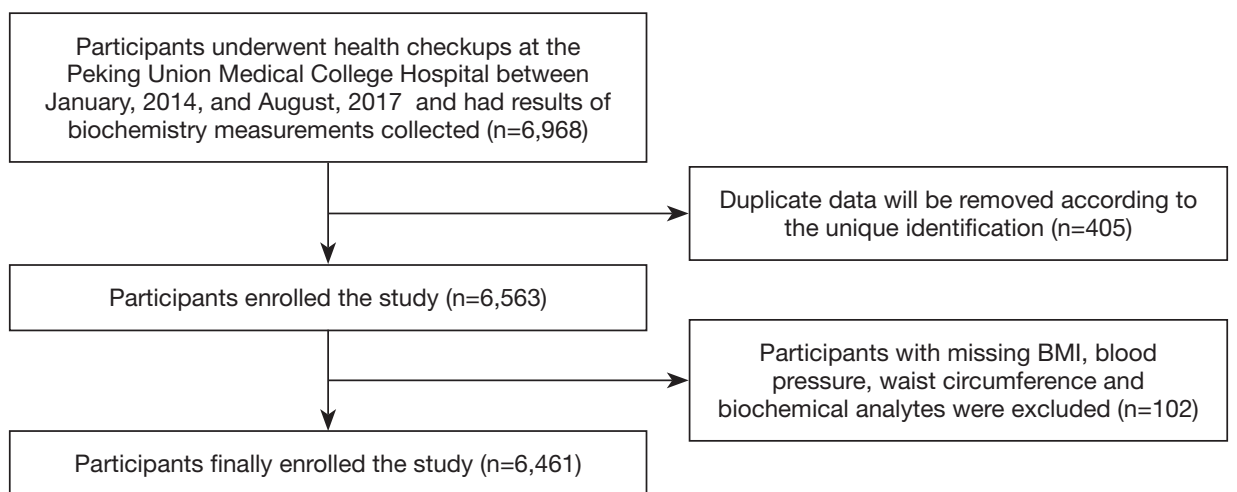

Figure 1 A schematic diagram showing the screening procedure and inclusion of study participants. The final sample size was 6,461 that consisted of 4,123 men and 2,338 women.

high-density lipoprotein cholesterol (HDL-C), low-density lipoprotein cholesterol (LDL-C), UA, creatinine (Cr), blood urea nitrogen (BUN), glutamyl transpeptidase (GGT), alanine aminotransferase (ALT), aspartate aminotransferase (AST), total bilirubin (TBil), and direct bilirubin (DBil) were measured. These common biochemical analytes were evaluated using a Beckman AU Series Automatic Biochemical Analyzer, and the specific reagents and calibrators required for each test. Baseline information of each subject such as body mass index (BMI), waistline (WC), systolic BP (SBP), and diastolic BP (DBP) was recorded. These evaluations were performed after excluding private information.

\section{ALDH2 rs671 polymorphism analysis}

To detect $A L D H 2$ rs671 polymorphism, genomic DNA was extracted from whole peripheral blood using the commercial whole blood genomic DNA extraction kit (Xi'an Tianlong Technology co. LTD, http://www.medtl.com/, Xi'an, China). ALDH2 rs671 polymorphism was detected using the $A L D H 2$ gene mutation detection kit, which relies on fluorescence quantitative PCR method (Beijing market gene technology co., Ltd., http://www.lepugene.com/). The fluorescence quantitative PCR reactions were manually performed according to the kit instructions to detect rs671 $A L D H 2$ polymorphism. Quality controls were prepared appropriately to ensure the accuracy of the results.

\section{Statistical analysis}

Statistical Package for the Social Sciences software, version
25.0 (IBM Inc., Armonk, NY, USA) was used to analyze the data. Kolmogorov-Smirnov tests and quantile-quantile plots were used to evaluate the distribution of the variables. If the variables followed normal distribution, they were described as the mean \pm standard deviation, whereas the data that did not meet normality hypothesis were presented as medians (1st quartile, $3 \mathrm{rd}$ quartile). For the data meeting normal distribution, a $t$-test was used for comparing the mean between the two groups. Univariate analysis of variance was used for comparing the mean between multiple groups, whereas Mann-Whitney $\mathrm{U}$ test and Kruskal-Wallis $\mathrm{H}$ test were used for data that did not fall within normal distribution. The least-significant-difference (LSD) and Student-Newman-Keuls (SNK) methods were used to perform multiple comparisons. The LSD method is the most sensitive of the multiple comparisons, whereas the SNK method strictly controls the total false positive error probability not exceeding $\alpha$. Therefore, the SNK method is the first choice when the results of the two methods are contradictory. Chi-square test was used for analyzing the differences in the comparisons of categorical data. Covariance analysis models were established for correcting the influencing factors. A $\mathrm{P}$ value of $<0.05$ was considered statistically significant.

\section{Results}

\section{Baseline information of subjects enrolled in the study}

A total of 6,461 subjects were included in this study, including 4,123 males and 2,338 females (Table 1). BMI, WC, SBP, DBP, Glu, TG, UA, Cr, BUN, GGT, ALT, AST, TBil and DBil levels were significantly higher in males 
Table 1 Baseline information of subjects enrolled the study

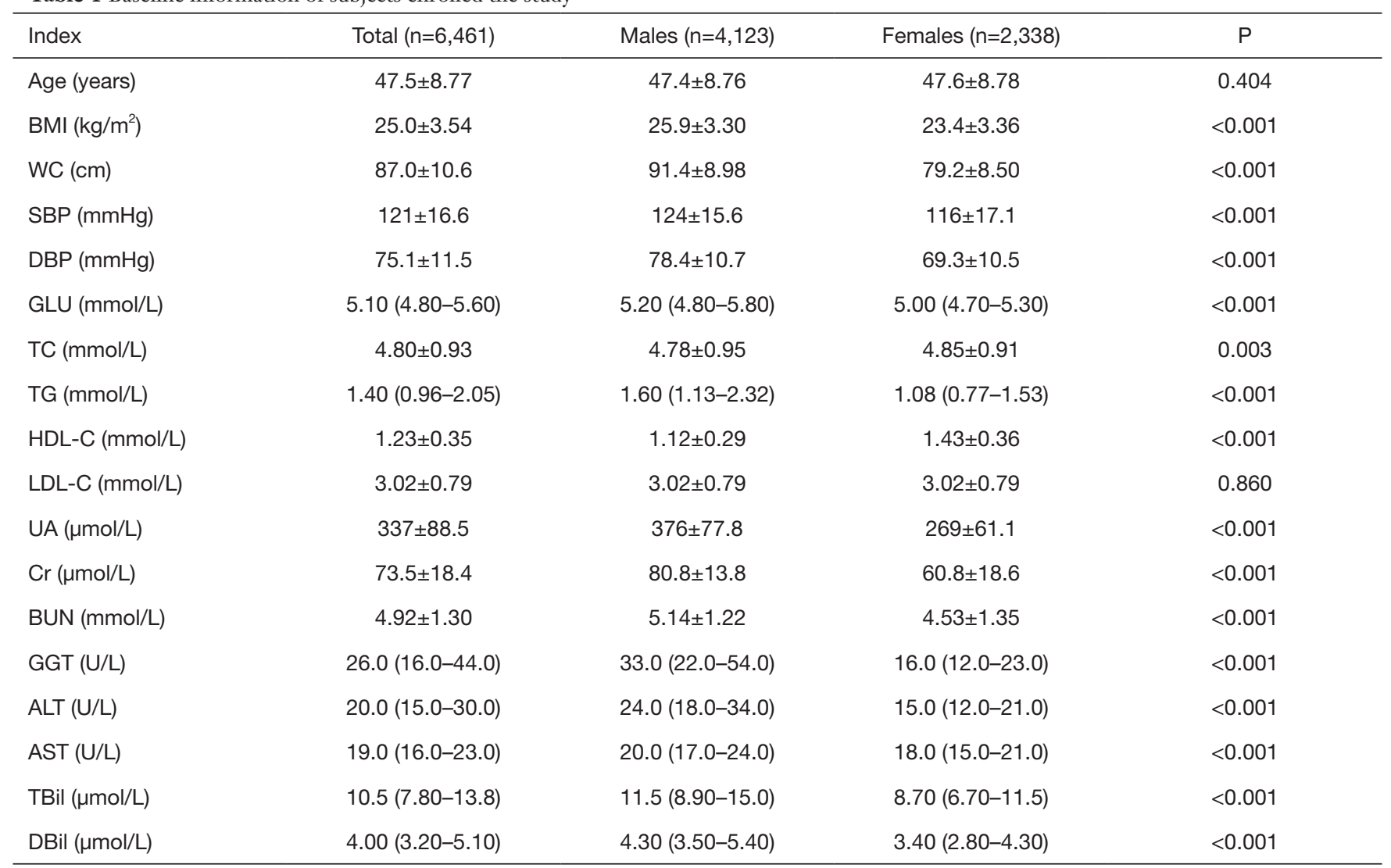

BMI, body mass index; WC, waistline; SBP, systolic blood pressure; DBP, diastolic blood pressure; GLU, glucose; TC, total cholesterol; TG, triglycerides; HDL-C, high-density lipoprotein cholesterol; LDL-C, low-density lipoprotein cholesterol; UA, uric acid; Cr, creatinine; BUN, blood urea nitrogen; GGT, glutamyl transpeptidase; ALT, alanine aminotransferase; AST, aspartate aminotransferase; TBil, total bilirubin; DBil, direct bilirubin; P, P value for differences in baseline information between females and males.

Table 2 Genotype and allele distribution of $A L D H 2$ rs671 in different genders

\begin{tabular}{|c|c|c|c|c|c|}
\hline Index & \multicolumn{3}{|c|}{ Genotype of $A L D H 2$ rs671, $\mathrm{n}(\%)$} & \multicolumn{2}{|c|}{ Allele of $A L D H 2$ rs671, n (\%) } \\
\hline Males & $2,781(67.5)$ & $1,227(29.8)$ & $115(2.8)$ & $6,789(82.3)$ & $1,457(17.7)$ \\
\hline Females & $1,619(69.2)$ & $666(28.5)$ & $53(2.3)$ & $3,904(83.5)$ & $772(16.5)$ \\
\hline Total & $4,400(68.1)$ & $1,893(29.3)$ & $168(2.6)$ & $10,693(82.8)$ & $2,229(17.2)$ \\
\hline
\end{tabular}

ALDH2, aldehyde dehydrogenase 2.

compared to females, while TC and HDL-C levels were significantly lower in females $(\mathrm{P}<0.05)$.

\section{Analysis of ALDH2 polymorphism}

The distribution of the three $A L D H 2$ rs671 genotypes is shown in Table 2. The G/G genotype was the most common, with 4,400 subjects accounting for $68.1 \%$ of the total subjects, followed by the G/A genotype, which was present in 1,893 individuals $(29.3 \%)$, and the A/A genotype being the least common, present in 168 subjects $(2.6 \%)$. The frequencies of the genotypes were in line with Hardy-Weinberg $(\mathrm{H}-\mathrm{W})$ equilibrium, indicating that the distribution of experimental data in each group was 
Table 3 The level of biochemical analytes and clinical characteristics in different genotypes of ALDH2

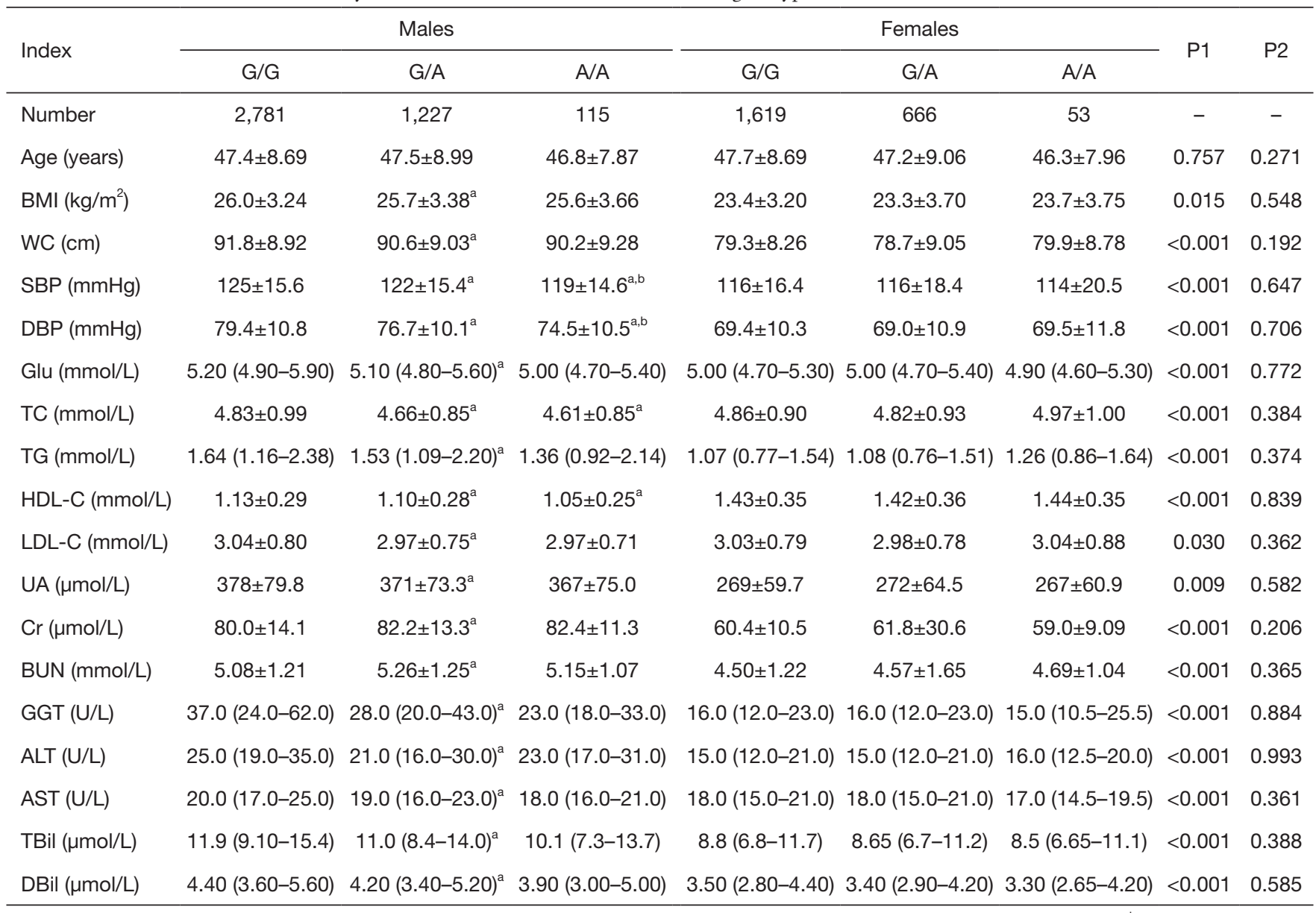

LSD and SNK were used for pairwise comparisons. ${ }^{a}$, when compared to G/G genotypes, P1 value was less than 0.05 ; ${ }^{b}$, when compared to the G/A genotype, the P2 value is less than 0.05. ALDH2, aldehyde dehydrogenase 2; BMI, body mass index; WC, waistline; SBP, systolic blood pressure; DBP, diastolic blood pressure; Glu, glucose; TC, total cholesterol; TG, triglycerides; HDL-C, high-density lipoprotein cholesterol; LDL-C, low-density lipoprotein cholesterol; UA, uric acid; Cr, creatinine; BUN, blood urea nitrogen; GGT, glutamyl transpeptidase; ALT, alanine aminotransferase; AST, aspartate aminotransferase; TBil, total bilirubin; DBil, direct bilirubin.

representative of the population. The chi-square test results showed that there was no significant difference in the distribution of the alleles and genotypes of $A L D H 2$ between males and females $(\mathrm{P}>0.05)$.

\section{Comparison of SUA levels and other biochemical analytes among ALDH2 rs671 genotypes}

The effect of each $A L D H 2$ genotype on the clinical characteristics and levels of biochemical analytes in males and females are presented in Table 3. In males, BMI, WC, SBP, DBP, Glu, TC, TG, HDL-C, LDL-C, UA, Cr, BUN, GGT, ALT, AST, TBil and DBil had significant differences between the different $A L D H 2$ genotypes $(\mathrm{P}<0.05)$.
Compared with the G/G genotype group, the BMI, WC, SBP, DBP, Glu, TC, TG, HDL-C, LDL-C, UA, GGT, ALT, AST, TBil and DBil levels in the G/A genotype group were significantly reduced, while the levels of $\mathrm{Cr}$ and BUN reflecting renal function were significantly increased $(\mathrm{P}<0.05)$. However, the difference of these parameters among the different genotypes of $A L D H 2$ in females was not statistically significant $(\mathrm{P}>0.05)$.

\section{Correlation between SUA level and ALDH2 rs671 genotypes and alleles}

In males, the different $A L D H 2$ genotypes $(\mathrm{P}=0.009)$ and alleles $(\mathrm{P}=0.002)$ were associated with SUA levels (Table 4). 
Table 4 SUA levels in different genotypes and alleles of ALDH2 in males

\begin{tabular}{|c|c|c|c|c|}
\hline Index & \multicolumn{2}{|c|}{ Before the adjustment } & \multicolumn{2}{|c|}{ After the adjustment ${ }^{a}$} \\
\hline ALDH2 genotype & & 0.009 & & 0.005 \\
\hline $\mathrm{G} / \mathrm{G}$ & $378 \pm 79.5^{b}$ & & $378 \pm 1.37^{b}$ & \\
\hline $\mathrm{G} / \mathrm{A}$ & $371 \pm 73.3$ & & $371 \pm 2.07$ & \\
\hline ALDH2 allele & & 0.002 & & 0.001 \\
\hline $\mathrm{G}(\mathrm{G} / \mathrm{G})$ & $378 \pm 79.5^{c}$ & & $378 \pm 1.37^{b}$ & \\
\hline$A(G / A+A / A)$ & $371 \pm 73.5$ & & $371 \pm 1.98$ & \\
\hline \multicolumn{5}{|c|}{$\begin{array}{l}\text { a , covariance analysis was conducted by correcting for factors such as } \mathrm{BMI}, \mathrm{Glu}, \mathrm{TC}, \mathrm{HDL}-\mathrm{C}, \mathrm{Cr} \text {, and GGT; }{ }^{\mathrm{b}} \text {, compared with } \mathrm{G} / \mathrm{A} \text { and } \\
\text { A/A genotypes, the difference in SUA level was statistically significant }(\mathrm{P}<0.05) \text {; }{ }^{\circ} \text {, compared with allele A, the difference of SUA was } \\
\text { statistically significant }(\mathrm{P}<0.05) \text {. SUA, serum uric acid; UA, uric acid; SD, standard deviation; ALDH2, aldehyde dehydrogenase } 2 \text {; } \\
\text { BMI, body mass index; Glu, glucose; TC, total cholesterol; HDL-C, high-density lipoprotein cholesterol; Cr, creatinine; GGT, glutamyl } \\
\text { transpeptidase. }\end{array}$} \\
\hline
\end{tabular}

The SUA levels for groups comprising the G/A and A/A genotypes and A-containing alleles were significantly lower than those of the $\mathrm{G} / \mathrm{G}$ genotypes and G-containing alleles $(\mathrm{P}=0.005)$. After adjusting for factors, such as BMI, Glu, TC, HDL-C, Cr, and GGT, the SUA levels of the different genotypes and alleles were still significantly different $(\mathrm{P}=0.001)$.

\section{Discussion}

$A L D H 2$ polymorphism is related to cardiovascular and cerebrovascular diseases, abnormal lipid metabolism, diabetes, metabolic syndrome, and Alzheimer's disease (7). Hyperuricemia is an independent risk factor for coronary artery disease, hypertension and obesity-related metabolic syndrome (8). In this study, we observed that the $A L D H 2$ rs671 genetic polymorphism correlated with the levels of SUA in males who underwent physical examination in Beijing, China, and the level of SUA was lower in males with an A allele than males with a $\mathrm{G}$ allele.

The distribution of $A L D H 2$ genotypes in the population showed significant racial specificity. A previous study reported that the frequency of the A allele at the $A L D H 2$ rs671 locus was very low in Caucasians, Africans (and their descendants), Papua New Guineans, Australian Aborigines, and Araucanians in South Chile, whereas its frequency was very high in the East Asian population, especially in the southeast Chinese population $(19,20)$. There are many ethnic groups in China, and the population markedly varies regionally. In this study, the distribution of $A L D H 2$ genotypes in Beijing, China, was analyzed. The G/G genotype was the most common, accounting for $68.1 \%$ of the total population, followed by the G/A genotype with $29.3 \%$. The A/A genotype is the least common, found in $2.6 \%$ of the population. The $A L D H 2$ variant frequencies in males from Shandong, China, were reported to be $80.5 \%$ for $G / G, 17.1 \%$ for $G / A$, and $2.4 \%$ for A/A (21). Even when the genotype distribution results of the male group in the present study were compared with those from the previous study that included males from Shandong, there were significant differences in the frequency of the $\mathrm{G} / \mathrm{G}$ and G/A genotypes (GG: $67.5 \%$ vs. $80.5 \%$; GA: $29.8 \%$ vs. $17.1 \%$ ). In addition, the $A L D H 2$ genotype frequency distribution was different in various regions of China: Xinjiang Province (22): GG: 67.1\%, GA: 29.8\%, AA: 3.1\%; Zhejiang Province (23): GG: 61\%, GA: 36\%, AA: 3\%. The results of genotype comparison in different regions of China suggest that the $A L D H 2$ genotype distribution may have regional differences. The different drinking cultures in different parts of China are a good example of the differences in results caused by regional genotypic differences.

In the present study, we observed that compared to $A L D H 2 \mathrm{G} / \mathrm{G}$ carriers, the levels of SBP, DBP, TC, TG, LDL-C, SUA, Cr, BUN, GGT, ALT, and AST were closer to the acceptable values in male $A L D H 2 \mathrm{G} / \mathrm{A}$ carriers. Previous studies have also reported that there is a relationship between $A L D H 2$ rs671 polymorphism 
and BP (24), lipid profile (21,24), liver function (24), and kidney function (24). Our results showing an association between $A L D H 2$ rs671 polymorphism and male lipid levels are consistent with the results reported by Han et al. Furthermore, the effects of $A L D H 2$ rs671 polymorphism on liver and kidney metabolites in males appear to be consistent with the findings of $\mathrm{Ma}$ et al.

A previous study reported that $A L D H 2$ polymorphism can affect SUA levels, and this SNP is significantly associated with the risk of gout (16). This study was performed in a Japanese population, and hence, whether their findings can be applicable to a Chinese population is unknown. Therefore, these results need to be confirmed by performing further studies. Our results showed that $A L D H 2$ polymorphism was significantly correlated with SUA level in males. Considering the potential effect of various factors such as BMI, and levels of Glu, TC, HDL-C, Cr, and GGT on SUA levels in this study, we further adjusted our data to account for these factors. Importantly, the correlation between $A L D H 2$ polymorphisms and SUA levels remained, which is consistent with the results of a previous study (25). This may be because those with the $A L D H 2$ A-containing allele show a decreased tendency toward alcohol consumption. ALDH2 A-containing allele carriers displayed lower alcohol consumption, likely due to discomfort caused by the accumulation of acetaldehyde, which can cause palpitations and nausea after ethanol consumption. ALDH2 G-containing allele carriers tended to consume alcohol, which leads to slower ATP production (13) and an increase in SUA level. However, these observations were not found in the women included in this study. A prospective study showed that only $2 \%$ $(6,245 / 302,510)$ of women drink alcohol in most weeks $(26)$. One possible reason why the SUA levels in females did not vary between the different genotypes may be because Han Chinese women generally do not drink alcohol or drink very little. Our results showing that the relationship between $A L D H 2$ genotype and SUA level was found only in males are consistent with those reported by a previous study (27).

Our study has several limitations. First, although the sample size of our study was large, our population is not representative of the whole of China. Second, the intensity of physical activity, dietary habits, smoking and drinking may also be associated with SUA level. To compensate for this limitation, we included several confounding variables in our multi-factor analysis such as GGT level and BP that are closely related to alcohol consumption. However, a larger multi-center study is still needed to better clarify the relationship between $A L D H 2$ polymorphism and UA metabolism.

\section{Conclusions}

This study is the first to show the distribution of $A L D H 2$ rs671 polymorphism in a healthy Chinese population in Beijing, and confirmed the gender-specific correlation between $A L D H 2$ polymorphism and SUA levels. Our study can also provide insight on the susceptibility and treatment strategies for SUA-associated diseases such as gout.

\section{Acknowledgments}

Funding: The study was supported by Capital's Funds for Health Improvement and Research (CFH-2020-1-4014); Beijing Key Clinical Specialty for Laboratory Medicine Excellent Project (No. ZK201000).

\section{Footnote}

Reporting Checklist: The authors have completed the MDAR reporting checklist. Available at http://dx.doi.org/10.21037/ atm-20-7113

Data Sharing Statement: Available at http://dx.doi. org/10.21037/atm-20-7113

Conflicts of Interest: All authors have completed the ICMJE uniform disclosure form (available at http://dx.doi. org/10.21037/atm-20-7113). The authors have no conflicts of interest to declare. The funding organization(s) played no role in the study design; in the collection, analysis, and interpretation of data; in the writing of the report; or in the decision to submit the report for publication.

Ethical Statement: The authors are accountable for all aspects of the work in ensuring that questions related to the accuracy or integrity of any part of the work are appropriately investigated and resolved. The study was conducted in accordance with the Helsinki Declaration (as revised in 2013) and was approved by the Ethics Committee of Peking Union Medical College \& Chinese Academy of Medical Sciences, Peking Union Medical College Hospital (ethical approval document number: S-K972). Since the data used in this study are removed privacy, it will not pose any risk to the subjects and cannot trace it back to subjects. 
Therefore, informed consent was not required.

Open Access Statement: This is an Open Access article distributed in accordance with the Creative Commons Attribution-NonCommercial-NoDerivs 4.0 International License (CC BY-NC-ND 4.0), which permits the noncommercial replication and distribution of the article with the strict proviso that no changes or edits are made and the original work is properly cited (including links to both the formal publication through the relevant DOI and the license). See: https://creativecommons.org/licenses/by-nc-nd/4.0/.

\section{References}

1. Singh JA, Reddy SG, Kundukulam J. Risk factors for gout and prevention: a systematic review of the literature. Curr Opin Rheumatol 2011;23:192-202.

2. Yuan $\mathrm{H}, \mathrm{Yu} \mathrm{C}, \mathrm{Li} X$, et al. Serum uric acid levels and risk of metabolic syndrome: a dose-response metaanalysis of prospective studies. J Clin Endocrinol Metab 2015;100:4198-207.

3. Kleber ME, Delgado G, Grammer TB, et al. Uric acid and cardiovascular events: A Mendelian randomization study. J Am Soc Nephrol 2015;26:2831-8.

4. Dehghan A, van Hoek M, Sijbrands EJ,et al. High serum uric acid as a novel risk factor for type 2 diabetes. Diabetes Care 2008;31:361-2.

5. Feig DI, Kang DH, Johnson RJ. Uric acid and cardiovascular risk. N Engl J Med 2008;359:1811-21.

6. Dehghan A, Kottgen A, Yang Q, et al. Association of three genetic loci with uric acid concentration and risk of gout: a genome-wide association study, Lancet 2008;372:1953-61.

7. Matsuo H, Yamamoto K, Nakaoka H, et al. Genomewide association study of clinically defined gout identifies multiple risk loci and its association with clinical subtypes. Ann Rheum Dis 2016;75:652-9.

8. Charles BA, Shriner D, Doumatey A, et al. A genome-wide association study of serum uric acid in African Americans. BMC Med Genomics 2011;4:17.

9. Mandal AK, Mount DB. The molecular physiology of uric acid homeostasis. Annu Rev Physiol 2015;77:323-45.

10. Yang B, Mo Z, Wu C, et al. A genome-wide association study identifies common variants influencing serum uric acid concentrations in a Chinese population. BMC Med Genomics 2014;7:10.

11. Wu J, Qiu L, Guo XZ, et al. Apolipoprotein E gene polymorphisms are associated with primary hyperuricemia in a Chinese population. PLoS One 2014;9:e110864.

12. Takeshita T, Morimoto K. Relationship between the $\mathrm{ADH} 2$ and ALDH2 genotypes and skin flushing after drinking alcohol. Nihon Rinsho 1997;55 Suppl:111-6.

13. Matsumoto A. The bidirectional effect of defective ALDH2 polymorphism and disease prevention. Adv Exp Med Biol 2019;1193:69-87.

14. Lu X, Wang L, Lin X, et al. Genome-wide association study in Chinese identifies novel loci for blood pressure and hypertension. Hum Mol Genet 2015;24:865-74.

15. Xu H, Zhang Y, Ren J. ALDH2 and stroke: A systematic review of the evidence. Adv Exp Med Biol 2019;1193:195-210.

16. Sakiyama M, Matsuo H, Nakaoka H, et al. Identification of rs671, a common variant of ALDH2, as a gout susceptibility locus. Sci Rep 2016;6:25360.

17. Zhang D, Yang $M$, Zhou D, et al. The polymorphism rs671 at ALDH2 associated with serum uric acid levels in Chinese Han males: A genome-wide association study. Gene 2018;651:62-9.

18. Kawamura Y, Nakaoka H, Nakayama A, et al. Genomewide association study revealed novel loci which aggravate asymptomatic hyperuricaemia into gout. Ann Rheum Dis 2019;78:1430-7.

19. Goedde HW, Agarwal DP, Fritze G, et al. Distribution of $\mathrm{ADH} 2$ and $\mathrm{ALDH} 2$ genotypes in different populations. Hum Genet 1992;88:344-6.

20. Li H, Borinskaya S, Yoshimura K, et al. Refined geographic distribution of the oriental ALDH2*504Lys (nee 487Lys) variant. Ann Hum Genet 2009;73:335-45.

21. Han S, Zhao X, Zhang X, et al. Acetaldehyde dehydrogenase 2 rs671 polymorphism affects hypertension susceptibility and lipid profiles in a Chinese Population. DNA Cell Biol 2019;38:962-8.

22. Jing CQ. Association between acetaldehyde dehydrogenase 2 gene rs671 polymorphism and essential hypertension in Han population from Xinjiang. Master's thesis. Urumqi: Xinjiang Medical University, 2015.

23. $\mathrm{Lv} \mathrm{YD}, \mathrm{Hu} \mathrm{XL}$, Wang $\mathrm{YZ}$, et al. A study on the association between gene polymorphism of ALDH2(Glu504Lys) and hypertension among Chinese Han people in Zhejiang. Preventive Medicine 2013;25:4-7.

24. Ma C, Yu B, Zhang W, et al. Associations between aldehyde dehydrogenase 2 (ALDH2) rs671 genetic polymorphisms, lifestyles and hypertension risk in Chinese Han people. Sci Rep 2017;7:11136. 
25. Chang B, Hao S, Zhang L, et al. Association between aldehyde dehydrogenase 2 Glu504Lys polymorphism and alcoholic liver disease. Am J Med Sci 2018;356:10-4.

26. Millwood IY, Walters RG, Mei XW, et al. Conventional and genetic evidence on alcohol and vascular disease

Cite this article as: $\mathrm{Wu} \mathrm{J}, \mathrm{Ma} \mathrm{C}$, Yi J, Chen Y, Ye A, Kong L, Qiu L, Xu T. Analysis of gender-specific associations between aldehyde dehydrogenase $2(A L D H 2)$ rs671 genetic polymorphisms and serum uric acid levels in Han Chinese. Ann Transl Med 2021;9(9):772. doi: 10.21037/atm-20-7113 aetiology: a prospective study of 500000 men and women in China. Lancet 2019;393:1831-42.

27. Jee YH, Jung KJ, Park YB, et al. Causal effect of alcohol consumption on hyperuricemia using a Mendelian randomization design. Int J Rheum Dis 2019;22:1912-9. 